\title{
Éjaculation provoquée par le vibromassage ou l'électrostimulation endorectale chez le blessé médullaire : injection intracytoplasmique de spermatozoïdes obtenus par stimulation endorectale chez neuf couples
}

\author{
G. EGON ${ }^{1}$, A. SCOBAI ${ }^{1}$, A.L. FERRAPIE ${ }^{1}$, D. DOMESSENT ${ }^{2}$, P. SIGOGNEAU2, S. GAY ${ }^{1}$ \\ 1Centre de l'Arche. Saint Saturnin, Le Mans ; 2Clinique du Tertre Rouge, Le Mans
}

\begin{abstract}
RÉSUMÉ
Moins de $15 \%$ des hommes avec une lésion complète de la moelle obtiennent une éjaculation lors de rapports sexuels ou de la masturbation. Le vibromassage du pénis permet d'obtenir une éjaculation pour au moins $70 \%$ d'entre eux. L'éjaculat recueilli au domicile par le couple est utilisé pour des auto-inséminations au moment le plus opportun du cycle. Les patients en échec du vibromassage obtiennent une éjaculation par stimulation endorectale dans la majorité des cas. Le stimulateur actuellement disponible est celui mis au point par SEAGER. Seule l'absence de spermatozoïde viable dans l'éjaculat conduit au prélèvement microchirurgical. L'échec des auto-inséminations, l'altération importante de la qualité du sperme chez ces blessés amènent fréquemment à la médicalisation de la procréation. Les neuf couples, à qui nous avons proposé une I.C.S.I. avec des spermatozoïdes obtenus par électrostimulation endorectale, ont mené à terme quatre grossesses.
\end{abstract}

Mots clés : blessés médullaires, électrostimulation, vibromassage, éjaculation, injection intracystoplasmique de spermatozoïdes,

\section{INTRODUCTION}

Le nombre de blessés médullaires est estimé à plus de 35000 en France et 1000 nouveaux cas surviennent chaque année. Quatre vingts pour cent des lésions concernent les hommes ; une majorité d'entre eux a entre 16 et 45 ans lors de l'accident.
L'anéjaculation dans les suites d'une lésion médullaire est un phénomène fréquent. Seulement, $15 \%$ des blessés médullaires peuvent obtenir une éjaculation lors d'un rapport sexuel ou par masturbation. Cette éjaculation est d'autant plus difficile à obtenir que la lésion est complète. L'anéjaculation est un motif fréquent de consultation pour les hommes blessés médullaires qui désirent procréer. L'altération de la qualité des spermatozoïdes vient s'ajouter aux difficultés de recueil.

Les deux techniques les plus utilisées pour obtenir une éjaculation que nous décrivons sont : le vibromassage du pénis et l'électrostimulation endorectale

\section{PHYSIOLOGIE DE L'EJACULATION}

L'éjaculation est contrôlée par trois centres médullaires :

- Le centre sympathique dorso-lombaire (TI1-L2) innerve par l'intermédiaire des nerfs hypogastriques, les canaux déférents, les vésicules séminales, la prostate, le col vésical. La contraction de ces organes responsable de la première phase de l'éjaculation entraîne l'émission de sperme dans l'urètre entre le col vésical fermé et le sphincter strié.

Correspondance :

Guy EGON, Centre de l'Arche, 72650. Saint Saturnin, Le Mans Tel 02.43.51.72.86. - Fax 02.43.51.72.57. - E. Mail : guyegon@hotmail.com

Communication à la Session SALF-AFU des $6^{\circ}$ Journées de la Fédération Française d'Étude de la Reproduction, Lyon, 5-7 septembre 2001 
- le centre parasympathique sacré (S2-S4) participe à l'innervation de la prostate. Il est partiellement responsable de la sécrétion du liquide séminal.

- le centre somatique sacré (S2-S4) innerve, par l'intermédiaire du nerf pudendal, les muscles bulbo-spongieux, ischio-caverneux et les muscles du plancher périnéal. La contraction de ces muscles, responsable de la deuxième phase de l'éjaculation, entraîne l'expulsion du sperme.

La stimulation des zones érogènes primaires (corpuscules de Krause-Finger sur la muqueuse du gland) ou secondaires, chemine par le nerf pudendal, les racines sacrées et la moelle jusqu'aux centres de l'éjaculation du cerveau (rhinencéphale). Les influx émis lors de la décharge des centres orgasmiques cérébraux cheminent par les voies motrices vers les centres médullaires de l'éjaculation, qui, chez l'homme normal, est concomitante de l'orgasme. Chez le blessé médullaire, la stimulation du gland lors de rapports sexuels ou lors de la masturbation ne permet que rarement d'obtenir une éjaculation non concomitante d'un orgasme.

\section{LE VIBROMASSAGE DU PENIS}

La stimulation mécanique par le vibromassage des corpuscules de Krause-Finger permet de déclencher le réflexe de l'éjaculation avec une coordination entre la phase d'émission et d'expulsion le plus souvent normale.

Cette technique a été décrite, pour la première fois, chez le paraplégique par Comar en 1970 [6] et plus largement diffusée par Brindley [3] en Europe et Francois [9] en France à partir de 1980.

Le vibromasseur « Ferti Care », actuellement commercialisé, a été conçu suite aux travaux de Sonksen [20]. Le réglage optimum est une amplitude de $2,5 \mathrm{~mm}$ et une fréquence de $100 \mathrm{~Hz}$.

Après vidange vésicale, le patient est confortablement installé en décubitus dorsal, buste relevé. Le disque de stimulation d'un diamètre de $35 \mathrm{~mm}$ est appliqué d'emblée au niveau du frein du prépuce rétracté, en regard du gland, en exerçant une pression assez forte sans changer de point de stimulation [8].

La durée maximale de la stimulation est de trois fois trois minutes avec une minute de repos entre chaque phase. L'éjaculation est annoncée par des contractures relativement importantes au niveau des abdominaux, puis des membres inférieurs. Elle est obtenue par voie antérograde, si le col vésical est compétent lors de la première phase de stimulation le plus fréquemment. En cas d'échec, un sondage est effectué pour rechercher une éjaculation rétrograde.

Les lésions locales cutanéo-muqueuses du gland sont rares : elles peuvent survenir lorsque l'application du vibreur a été longue. Elles ne nécessitent pas de traitement spécifique, en dehors de l'arrêt du vibromassage jusqu'à guérison.

Lorsque la lésion est au-dessus de T12 et qu'elle respecte les deux centres de l'éjaculation, le taux de succès du vibromassage est supérieur à $80 \%$. Lorsque les centres de l'éjaculation sont dans le territoire lésionnel, des succès sont possibles si la lésion est incomplète.

Compte tenu de ces résultats, le vibromassage est proposé en première intention à tous les patients blessés médullaires décrivant une anéjaculation. Pour les patients en situation d'échec, on utilise l'électrostimulation endorectale.

\section{L'ELECTROSTIMULATION ENDORECTALE}

Cette technique, d'abord utilisée en médecine vétérinaire, a été introduite chez l'homme en 1948 par Horne [12].

\section{Matériel}

\section{a) Technique de Brindley}

En 1984, Brindley [4] rapporte un pourcentage d'éjaculation antérograde ou rétrograde de $60 \%$ à partir d'une série de 84 patients : il obtient 11 grossesses et 9 naissances.

La technique de Brindley consiste à stimuler, avec un doigtier moulé à la taille de l'index, en matière thermoformable sur lequel sont disposées une cathode de stimulation d'un diamètre de $8 \mathrm{~mm}$ et deux anodes d'un même diamètre, directement à travers la paroi du rectum, le nerf hypogastrique droit, puis gauche. Les paramètres du courant délivré par le stimulateur sont les suivants : durée de l'impulsion $100 \mathrm{~ms}$, fréquence $15 \mathrm{~Hz}$, voltage 9 à 108 volts. On obtient une éjaculation chez tous les patients, dont le syndrome lésionnel médullaire épargne, au moins partiellement, les centres sympathiques dorso-lombaires.

\section{b) Technique de Seager}

En 1987, Halstead [10] décrit une technique de stimulation endorectale différente, déjà utilisée par Seager [18] depuis de nombreuses années en médecine vétérinaire. Un courant alternatif sinusoïdal délivré par l'intermédiaire d'une sonde rectale d'un diamètre de $30 \mathrm{~mm}$ permet de stimuler, à travers la paroi rectale, les organes de l'éjaculation (vésicules séminales, canaux déférents, prostate). Heruti [11] rapporte dans une publication récente une émission de sperme chez $100 \%$ des 84 patients traités, quel que soit le niveau de la lésion médullaire avec présence de spermatozoïdes pour 74 d'entre eux. Le matériel de stimulation mis au point par Seager [19] est actuellement le seul disponible.

\section{Méthode}

a) Technique de Brindley 
Le patient, vessie et rectum vides, est installé en décubitus dorsal, en position gynécologique. On introduit précautionneusement le doigtier de stimulation lubrifié avec un gel aqueux dans le canal anal. L'électrode de complément introduite, on stimule latéralement les fibres sympathiques myélinisées au niveau du "point obturateur". L'émission de sperme survient dans un délai de 5 à 30 secondes. En cas d'échec, on stimule le point contro-latéral en changeant de main. La tension artérielle est prise avant le début de la stimulation et avant la stimulation contro-latérale. Une hyperréflectivité autonome naissante cède le plus souvent dès l'arrêt de la stimulation. La fréquence de stimulation utilisée est de $15 \mathrm{~Hz}$ avec un courant de 54 volts ; 80 ou 90 volts sont quelquefois nécessaires. Le sperme recueilli est envoyé au laboratoire. En cas d'échec ou de faible volume émis, un sondage est effectué. Un succès est considéré comme rétrograde si l'urine contient au moins 5 millions de spermatozoïdes. L'éjaculation est souvent obtenue sans érection.

\section{b) Technique de Seager}

Après avoir vidé la vessie et l'ampoule rectale, le patient est confortablement installé en décubitus latéral droit. La sonde rectale, d'un diamètre choisi en fonction de la taille du sphincter anal, est introduite en regard de la prostate et des vésicules séminales à travers la paroi rectale.

L'éjaculation est obtenue dans un délai de 5 à 15 minutes, avec un courant alternatif sinusoïdal d'une intensité de 100 à 400 mas et une tension de 4 à 10 volts pour les lésions basses.

\section{COMPLICATIONS SPECIFIQUES AU VIBROMASSAGE ET A L'ELECTROSTIMU- LATION ENDORECTALE}

Le vibromassage et l'électrostimulation endorectale doivent s'accompagner d'un monitorage de la tension artérielle chez tous les blessés médullaires, dont le niveau lésionnel est au-dessus de T6. Ces deux techniques peuvent entraîner une hyperréflectivité autonome, responsable de céphalées et surtout d'une augmentation dangereuse de la pression artérielle. Un traitement curatif ou préventif par nifédipine, en cas d'antécédents connus d'hyperréflectivité autonome, est institué.

\section{TÉCHNIQUES DE PROCRÉATION}

\section{L'auto-insémination au domicile par le couple}

Lorsque l'éjaculation est obtenue par le vibromassage et que la qualité du sperme est acceptable, l'auto-insémination avec une seringue au moment le plus opportun du cycle est enseignée au couple. Plusieurs grossesses ont été rapportées selon cette technique $[3,7,14,15,21]$

\section{Procréation assistée}

L'échec de l'auto-insémination au domicile, l'échec du vibromassage nécessitant le recours à l'électrostimulation endorectale, conduisent à médicaliser la procréation. Le sperme recueilli est utilisé pour une insémination intra-utérine ou une fécondation in vitro en fonction du nombre de spermatozoïdes mobiles. Compte tenu de l'altération majeure de la qualité du sperme et des difficultés de recueil par électrostimulation (éjaculation partiellement ou totalement rétrograde), l'injection intracytoplasmique d'un spermatozoïde est largement utilisée pour ces couples.

Nous rapportons les résultats d'une fécondation in vitro avec I.C.S.I. chez 9 couples, dont l'homme présente une lésion médullaire.

\section{a) Patients et méthodes}

Neuf hommes blessés médullaires (4 tétraplégiques, 5 paraplégiques), âgés de 28 à 38 ans vivant en couple depuis plus de 2 ans, ont subi 13 électroéjaculations. Aucun n'obtient d'éjaculation par vibromassage. Nous avons pratiqué cette électroéjaculation sous anesthésie générale, associée à une courte curarisation (celocurine) pour éviter l'émission rétrograde de sperme.

\section{b) Résultats}

L'épargne, au moins partielle du centre sympathique dorsolombaire, a permis d'obtenir un éjaculat dans tous les cas.

Les spermogrammes montrent une altération importante de la mobilité et du nombre des spermatozoïdes, alors que le volume de l'éjaculat est normal (Tableau 1).

Nous avons injecté 118 ovocytes et obtenu 74 embryons ( $60 \%) .42$ embryons, dont 4 congelés ont été transférés sur 16 cycles.

Quatre grossesses menées à terme ont conduit à la naissance de 5 enfants. Un couple a eu 3 enfants en 2 cycles d'I.C.S.I., dont un à partir de deux embryons congelés. Un autre couple a eu un enfant à partir de sperme congelé. Le taux de naissance par cycle est de $25 \%$ et le taux de naissance par couple est de $33 \%$.

Tableau 1 : Caractéristiques du sperme obtenu par electroéjaculation.

\begin{tabular}{lccc}
\hline & volume $(\boldsymbol{m l})$ & $\begin{array}{l}\text { nbre de } \\
\text { spzoïdes/ml }\end{array}$ & $\begin{array}{l}\text { mobilité } \\
(\%)\end{array}$ \\
\hline $\begin{array}{l}\text { tétraplégiques } \\
\begin{array}{l}\text { 2 paraplégiques } \\
\text { au-dessous de T10 }\end{array}\end{array}$ & 2,8 & $41,6 \mathrm{M}$ & 7,6 \\
$\begin{array}{l}\text { 3 paraplégiques } \\
\text { au-dessus de T10 }\end{array}$ & 4 & $21 \mathrm{M}$ & 10,3 \\
\hline
\end{tabular}




\section{c) Discussion}

Dans notre série, les 9 hommes ne peuvent pas obtenir d'éjaculation par vibromassage. L'altération importante de la qualité du sperme obtenu par électrostimulation nous conduit à proposer une injection intracytoplasmique d'un spermatozoïde selon la technique classiquement décrite.

L'altération de la qualité du sperme chez le blessé médullaire est un phénomène constant décrit dans toutes les publications $[13,15,16]$. L'électroéjaculation sous anesthésie générale associée à une courte curarisation permet d'obtenir un éjaculat presque totalement antérograde : les volumes que nous avons obtenus sont supérieurs à ceux décrits dans la littérature [2].

Schatte [17] rapporte à propos de 17 couples, dont le sperme a été recueilli par électrostimulation, pour une fécondation par I.C.S.I., un taux de grossesse de $15 \%$ par cycle et de $26 \%$ par couple. Seuls 10 hommes sur les 17 de sa série présentent une lésion médullaire.

\section{CONCLUSION}

Le vibromassage du pénis et l'électrostimulation endorectale permettent d'obtenir une éjaculation ou une émission de sperme chez presque $100 \%$ des blessés médullaires. Nous conseillons d'utiliser chez tous les patients le vibromassage du pénis, qui se fera à domicile après apprentissage.

Nous réservons l'électrostimulation endorectale aux échecs du vibromassage. Seule l'absence de spermatozoïde viable dans l'éjaculat conduit au prélèvement micro-chirurgical. La difficulté de recueil par électrostimulation d'un sperme très altéré peut conduire à l'utilisation de l'injection intracytoplasmique d'un spermatozoïde.

\section{RÉFÉRENCES}

1. BENNETT C.J. et al. : Electroejaculation and assisted reproductive techniques for anejaculatory infertility. Obstet. Gynecol., $1996,87: 22-26$

2. BIERING-SORENSEN F, SONKSEN J. : Sexual function in spinal cord lesioned men. Spinal Cord, 2001, $39: 455-470$

3. BRINDLEY G.S. : The fertility of men with spinal injuries. Paraplégia, 1984, 22 : 337-348

4. BRINDLEY G.S.: Electroejaculation : its techniques neurological implications and use. J. Neurol. Neurosurg. Psychiatry, 1981, 44: 9-18
5. CHUNG PH et al. : Assisted fertility using electroejaculation in men with spinal cord injury - a review of literature. Fertil. Steril., $1995 ; 64: 1-9$

6. COMARR A.E. : Sexual function among patients with spinal cord injury. Urol. Int., 1970, $25: 134$

7. DAHLBERG A., RUUTU M, HOVATTA O. : Pregnancy results from a vibrator application, electroejaculation, and vas aspiration program in spinal cord injured men. Hum. Reprod., 1995, 10 ; 2305-2307

8. EGON G. et al : Ejaculation provoquée chez le paraplégique. Andrologie, 1994, 4 : 160-168

9. FRANCOIS N., LICHTENBERGER J.M., JOUANNET P et al : L'éjaculation par le vibromassage chez le paraplégique : à propos de 50 cas avec 7 grossesses. Ann. Med. Phys., $1980: 23-24$

10. HALSTEAD L.S., VERVOORT S; SEAGER S. W. J. : Rectal probe électrostimulation in the treatment of inejaculatory spinal cord injured men. Paraplégia, 1987, $25: 120-129$

11. HERUTI R. J et al : Treatment of male infertility due to spinal cord injury using rectal probe electroejaculation : the Israeli experience. Spinal Cord, 2001, $39:$ 168-175

12. HORNE H. W., PAULL D. P. , MUNRO, D. : Fertility studies in human male with traumatic injuries of the spinal cord and cauda equina. N. Engl. J. Med, 1948. 239 : 959-961

13. LUCAS M.G. et al : Sperm retrieval by electroejaculation. Preliminary experience in patients with secondary anejaculation. Br. J. Urol., 1991, 67 : 191-194

14. LOCHNER-ERNST D. et al : Conservative and surgical semen retrieval in patients with spinal cord injury. Spinal Cord, 1997, 35 : 463-468

15. NEHRA A. et al. : Vibratory stimulation and rectal probe electroejaculation as therapy for patients with spinal cord injury : semen parameters and pregnancy rates. J. Urol., 1996, 155 : 554559

16. OHL D.A. et al. : Predictors of success in electroejaculation of spinal cord injured man. J. Urol 1993, $149: 1350-1354$

17. SCHATTE E. C. et al. : Treatment of infertility due to anejaculation in the male with electroejaculation and intracytoplasmic sperm injection. J. Urol., 2000, $163: 1717-1720$

18. SEAGER S.W.J. : The Breeding of captive wild species by artificial methods. Zoo. Biol., 1983, 2 : 235

19. SEAGER S.W.J., DVM , LAURO S. HALSTEAD MD : Fertility options and success after spinal cord injury. Spinal Cord, 1993, 20, 543-548

20. SONKSEN M. D. et al : Ejaculation induced by penile vibratory stimulation in men with spinal cord injuries. The importance of the vibratory amplitude. Paraplegia, 1994, 32 : 651-660

21. SONKSEN J. et al : Pregnancies after assisted ejaculation procedures in men with spinal cord injuries. Arch. Phys. Med. Rehabil., 1997,$78 ; 1059-1061$ 


\begin{abstract}
Ejaculation induced by penile vibratory stimulation or rectal probe electrostimulation in the treatment of anejaculatory spinal cord injured men : the use of intracytoplasmic sperm injection with electroejaculates in 9 couples
\end{abstract}

G. EGON, A. SCOBAI, A.L. FERRAPIE, D. DOMESSENT, P. SIGOGNEAU, S. GAY

800 to 1,000 cases of traumatic spinal cord injury (S.C.I.) are observed in France each year. At present time, there are more than 35,000 survivors of S.C.I. in France. Studies show that $80 \%$ of injuries occur in men and $82 \%$ occur in individuals between the ages of 16 and 45 years. It is well known that more than $80 \%$ of men with S.C.I. suffer from ejaculation dysfunction. Since most of these couples are young, many desire a family and seek help to remedy their infertility. Two methods are mainly used to retrieve sperm from S.C.I. men: penile vibratory stimulation and rectal electrostimulation.

Penile vibratory stimulation to induce ejaculation is recommended as first-line treatment, and S.C.I. men should only be referred for electroejaculation after failure of this technique.

More than $\mathbf{7 0} \%$ of patients respond to penile vibratory stimulation with anterograde ejaculation. Patients who fail to ejaculate by penile vibratory stimulation are treated by electroejaculation.

Their ejaculates often have normal sperm counts, but with a higher proportion of immotile sperm than in men without S.C.I. The authors report the pregnancy outcome of a series of 9 couples undergoing combined electroejaculation and in vitro fertilization with intracytoplasmic sperm injection: $25 \%$ pregnancies per cycle and $33 \%$ pregnancies per couple.

Key-words: spinal cord injury, electrostimulation, vibration, ejaculation, intracystoplasmic sperm injection. 